\title{
Guest Editorial: Selected Papers from the First Joint Israeli-Turkish Workshop on Nanophotonics
}

\author{
Zeev Zalevsky \\ School of Engineering, \\ Bar-Ilan University, \\ Ramat-Gan 52900, Israel \\ zalvesz@eng.biu.ac.il \\ Ekmel Ozbay \\ Nanotechnology Research Center (NANOTAM), \\ Bilkent University Bilkent, \\ Ankara 06800, Turkey \\ ozbay@bilkent.edu.tr

\section{Ibrahim Abdulhalim} \\ Electro-optics Engineering Program, \\ Ben-Gurion University of the Negev, \\ Beer-Sheva 84105, Israel \\ abdulhlm@bgu.ac.il
}

Nanophotonics is one of the most appealing fields, having a large variety of academic as well industrial applications. The main advantage of this field is in the prospects of devices with increased multifunctionality, higher level of integration, faster operation rate, lower power consumption, small size, and low cost. Nanophotonic devices can be used as building blocks in processing chips delivering ultrafast operation rate, reduced power consumption, lower noise level due to improved confinement of photons, and increased capability for transmission of larger bandwidths of information. Nanophotonics would help in realizing in-fiber light sources with controllable properties. Nanophotonics could be exploited in constructing a laboratory-on-a-chip that can extract all the required physiological data from a single drop of blood from a patient. Nanophotonic concepts can be used for designing spatial masks that may assist in bending the conventional laws of diffraction and thus overcoming the Rayleigh limit for spatial resolution in imaging systems. In addition, this field has significantly increased academic as well as industrial potentials due to the enormous gains achieved in the last decade in fabrication capabilities.

Israel and Turkey are almost geographical neighbors. In both countries, nanophotonics researchers have recorded impressive academic as well as industrial achievements. On March 30, 2007, in Bar-Ilan University (Israel) the first joint Israeli-Turkish workshop on nanophotonics was held. The purpose of this workshop was to generate close binational collaboration links between Israeli and Turkish scientists, research facilities and institutes, as well as industries and ministries. In this workshop, leading Turkish and Israeli scientists presented their nanophotonics-related research work. This special section of the Journal of Nanophotonics contains a selection from the papers that were presented at the workshop. We, the Guest Editors of this special section, hope that a joint Israeli-Turkish workshop shall become an annual event. 